\title{
Cerebral infarctions as manifestation of ovarian clear cell carcinoma: report of two cases and review of the literature
}

\author{
Hirokazu Naoi · Hiroyuki Hashimoto • Etsuko Kajimoto • \\ Masumi Takeda - Susumu Yoshida $\cdot$ Takashi Miyatake • \\ Takeshi Yokoi $\cdot$ Shigeyuki Isaka $\cdot$ Masaaki Nagamatsu
}

Received: 9 December 2012 / Accepted: 20 February 2013/Published online: 10 March 2013

(C) The Japan Society of Clinical Oncology 2013

\begin{abstract}
Venous thromboembolic events (VTE) may occur before the diagnosis of cancer, and VTE can be a predictor of the subsequent diagnosis of cancer. Stroke as well as VTE may be the first manifestation of a subsequent diagnosis of cancer. We report two cases of patients showing cerebral infarction as the first manifestation of clear cell carcinoma of the ovary. The first case is a 55-year-old woman, who had no significant past medical history, lost her consciousness and was diagnosed with bilateral thalamic infarction according to magnetic resonance imaging results. After the diagnosis of infarction, advanced ovarian clear cell carcinoma was diagnosed at the interval of 4 months. Although an operation for ovarian cancer and 6 cycles of subsequent chemotherapies were executed, a recurrent disease occurred in the abdominal cavity. There also were relapses of infarction accompanied by ovarian cancer progression, and she died of ovarian cancer. The second case was a 62-year-old woman, who had repeated infarction episodes, and at the second infarction episode, her respiratory state also worsened. Computed tomography demonstrated swelling of her left ovary, venous thrombosis in the left femoral vein, and pulmonary arterial embolism. An operation for ovarian cancer was performed and early stage clear cell adenocarcinoma of the left ovary was confirmed by pathology. She has remained without evidence of recurrent cancer for 2 years after the completion of chemotherapy. Once cerebral infarction is diagnosed, a comprehensive search for unknown malignant disease should be performed, particularly ovarian clear cell carcinoma.
\end{abstract}

H. Naoi · H. Hashimoto $(\bowtie) \cdot$ E. Kajimoto $\cdot$ M. Takeda . S. Yoshida - T. Miyatake - T. Yokoi - S. Isaka - M. Nagamatsu Kaizuka City Hospital, 3-10-20 Hori, Kaizuka, Osaka 5970015, Japan

e-mail: jolly@pc4.so-net.ne.jp
Keywords Cerebral infarction - Manifestation . Ovarian clear cell carcinoma $\cdot$ Review

\section{Introduction}

The association between venous thromboembolic events (VTE) and malignant disease is well known, and ovarian cancer, in particular, is associated with high rates of coexistent VTE [1]. Among epithelial ovarian tumors, histologically, clear cell carcinoma shows a strong association with deep venous thrombosis (DVT) and pulmonary embolism (PE) [2-4].

Occasionally, VTE symptoms are recognized prior to the diagnosis of malignant disease, and VTE can be a predictor of the subsequent diagnosis of cancer, especially pancreas, ovary, liver, and brain cancers [5-7].

Stroke as well as VTE may be the first manifestation of ovarian cancer. Although there is no study about the incidence of cerebral infarction before the diagnosis of ovarian cancer, several reports show that cerebral infarctions in patients with ovarian cancer may be caused by nonbacterial thrombotic endocarditis or by a hypercoagulable state [812].

Here we report two cases of patients showing cerebral infarction as the first manifestation of clear cell carcinoma of the ovary.

\section{Case 1}

A 55-year-old woman, who did not have significant past medical history, suddenly lost her consciousness and was diagnosed with bilateral thalamic infarction according to magnetic resonance imaging (MRI) results (Fig. 1). Her 


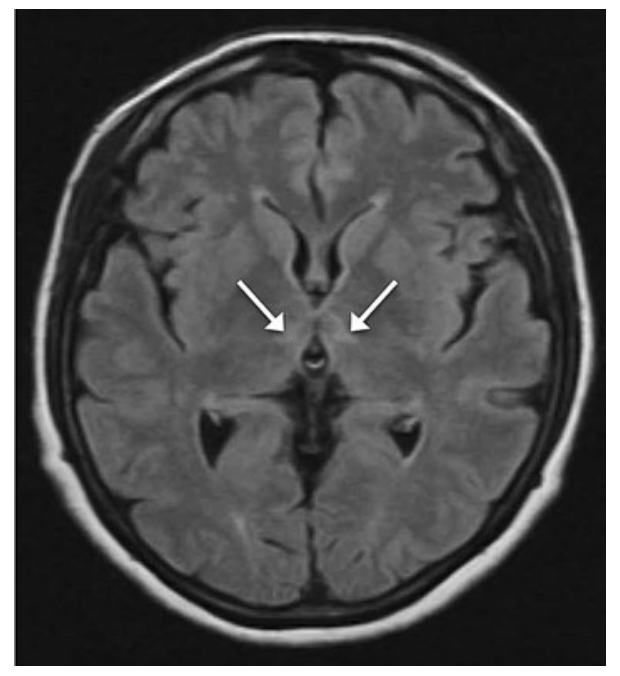

Fig. 1 Axial T2-weighted brain MRI. Arrows show enhancement in bilateral thalamus representing old infarct

consciousness has recovered on the next day without any medication. Transthoracic echocardiography (TTE) for detection of intracardiac sources of cerebral emboli did not reveal any abnormal findings. Laboratory data of platelet counts, fibrin degradation products (FDP), fibrinogen, lupus anticoagulant, anticardiolipin, antithrombin III, protein C, protein $\mathrm{S}$, and antinuclear antibody were all unremarkable. The patient was discharged without any antiplatelet and anticoagulant agents. Four months later, she developed abdominal pain. Pelvic MRI demonstrated swelling of her right ovary and disseminated lesions on the peritoneum (Fig. 2a, b). Advanced ovarian cancer was suspected, and an operation was performed. Metastatic lesions were found on the omentum and the transverse and descending colon. Total hysterectomy, bilateral salpingo-oophorectomy, omentectomy, pelvic lymph node dissection, and sigmoidectomy were all performed. She was optimally debulked with exploration. After surgery, low molecular weight heparinoid (Orgaran) was administered by subcutaneous injection in a dose of 750 anti-Factor Xa units every $12 \mathrm{~h}$ for 2 days. The pathology was confirmed to be clear cell carcinoma of the right ovary. The FIGO stage was IIIc, and six cycles of postoperative adjuvant chemotherapy with carboplatin $($ AUC $=6)$ and paclitaxel $\left(175 \mathrm{mg} / \mathrm{m}^{2}\right)$ every 3 weeks were completed. There was no sign of recurrent cancer after the chemotherapy and the patient was discharged. Three months later after the completion of chemotherapy, she felt distension in the abdomen. Massive ascites and disseminated cancer lesions in the abdominal cavity were detected with computed tomography (CT). Chemotherapy for recurrent cancer with irinotecan $\left(60 \mathrm{mg} / \mathrm{m}^{2}\right.$, days 1,8 , and 15$)$ and cisplatin $\left(60 \mathrm{mg} / \mathrm{m}^{2}\right.$, day 1$)$ was started.

One week after starting chemotherapy, hemiparesis of the right side and unconsciousness appeared. Magnetic
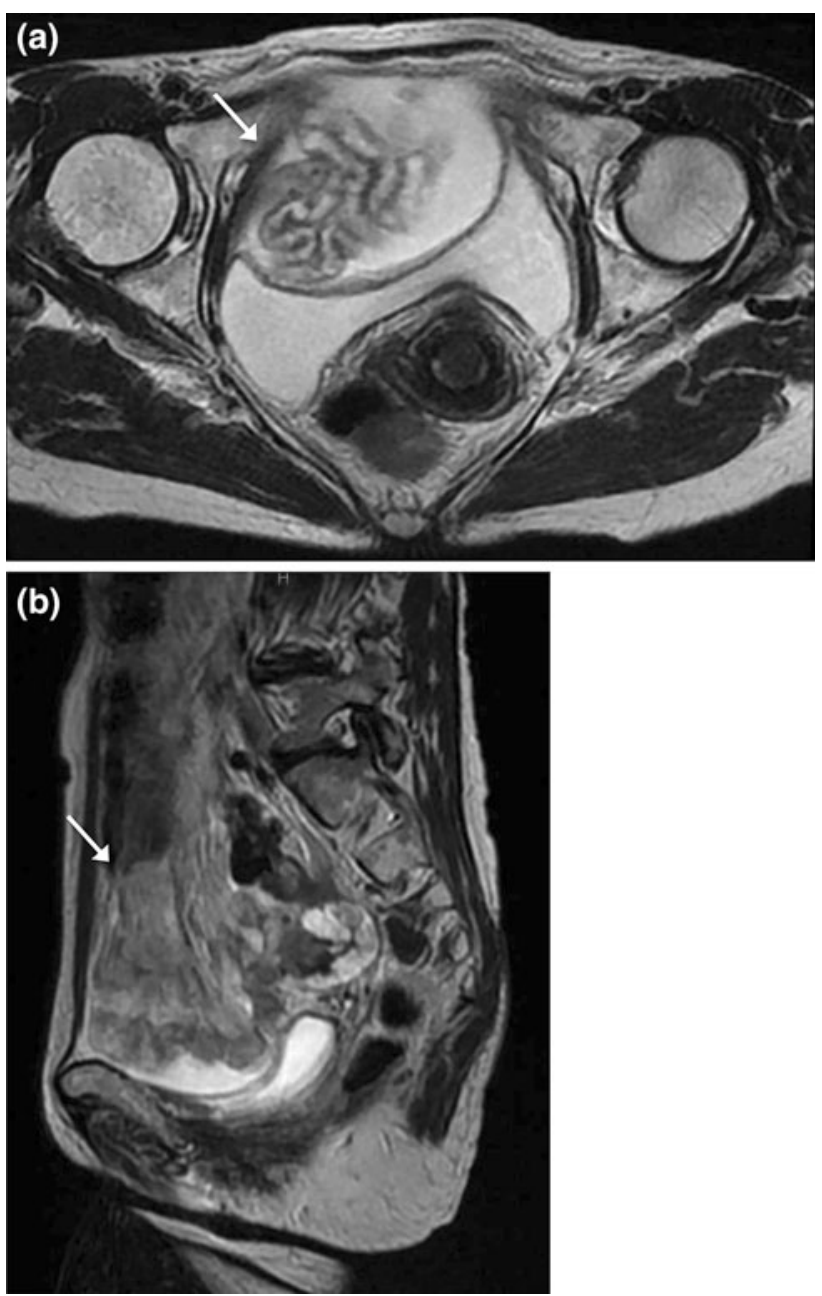

Fig. 2 a Axial and b sagittal T2-weighted pelvic MRI show a cystic and solid mass

resonance angiography showed infarction in the right middle cerebral artery.

Laboratory examinations revealed slightly decreased platelet counts and elevation of FDP. Fibrinogen level, prothrombin time (PT), and activated partial thromboplastin time (APTT) were within the normal range. A CT scan did not detect any DVT in the abdomen or pelvis, or PE. Then, anticoagulant therapy with intravenous heparin (10,000 U/day) was started and the symptoms of hemiparesis and unconsciousness recovered temporarily; however, relapses of infarction occurred repeatedly after the episode, and she died of progression of recurrent ovarian cancer.

\section{Case 2}

A 62-year-old woman with hypertension and diabetes mellitus showed the symptoms of inarticulate speaking and hemiparesis of the left side. MRI confirmed the diagnosis of infarction at the right frontal lobe and left cerebellum 


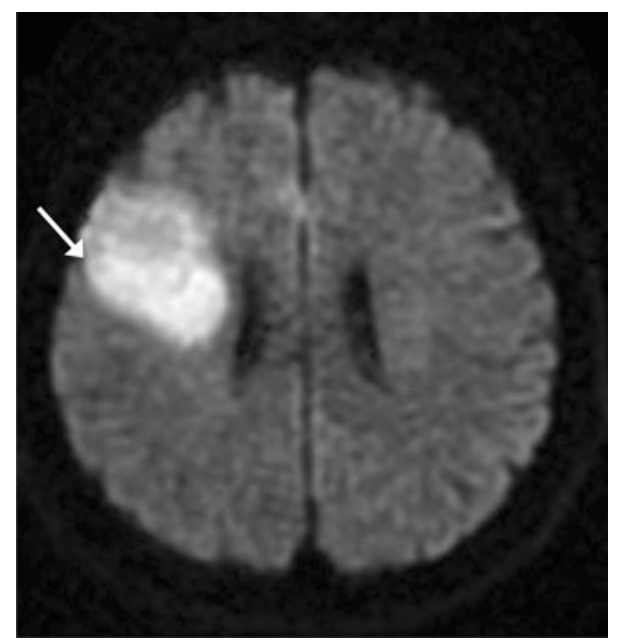

Fig. 3 Axial diffusion-weighted brain MRI shows an infarct of right MCA territory

(Fig. 3). Platelet counts, FDP, and fibrinogen level were normal. Her consciousness was clear and therefore she underwent antiplatelet therapy with aspirin (100 mg/day) and cilostazol (200 mg/day). The symptoms disappeared and she was discharged. Three months later, the inarticulate speech and left-sided hemiparesis developed again; this time MRI showed an obstruction in the right middle cerebral artery (MCA). Repeated transthoracic echocardiogram (TTE) for the detection of sources of cerebral emboli did not show any irregular sign and multiple blood cultures searching for the sign of endocarditis remained sterile. Laboratory examinations revealed normal platelet counts and mild elevation of FDP and fibrinogen level. Lupus anticoagulant, anticardiolipin, antithrombin III, protein $\mathrm{C}$, protein $\mathrm{S}$, and antinuclear antibody were normal. She underwent anticoagulant therapy with combination of intra-thrombus rtPA and intravenous heparin to achieve therapeutic anticoagulation. During this hospitalization, her respiratory status worsened. CT demonstrated swelling of the left ovary $(20 \mathrm{~cm}$ at the diameter), thrombosis in the left femoral vein, and pulmonary arterial embolism. Pelvic MRI showed an intra-pelvic mass with a mixture of cystic and solid components (Fig. 4), which indicated an ovarian cancer. After installation of a filter in the inferior vena cava to prevent $\mathrm{PE}$, an operation for intra-pelvic mass was performed. The left ovary was swollen and adhered rigidly and extensively to the small intestine. Total hysterectomy, bilateral salpingo-oophorectomy, and omentectomy were performed. On the intraoperative inspection, the other intra-abdominal organs were grossly tumor free. The pathology confirmed the diagnosis of clear cell adenocarcinoma. Aggressive surgical exploration was not performed to avoid a long operation time, because of her active thrombotic status. The FIGO stage was Ic.
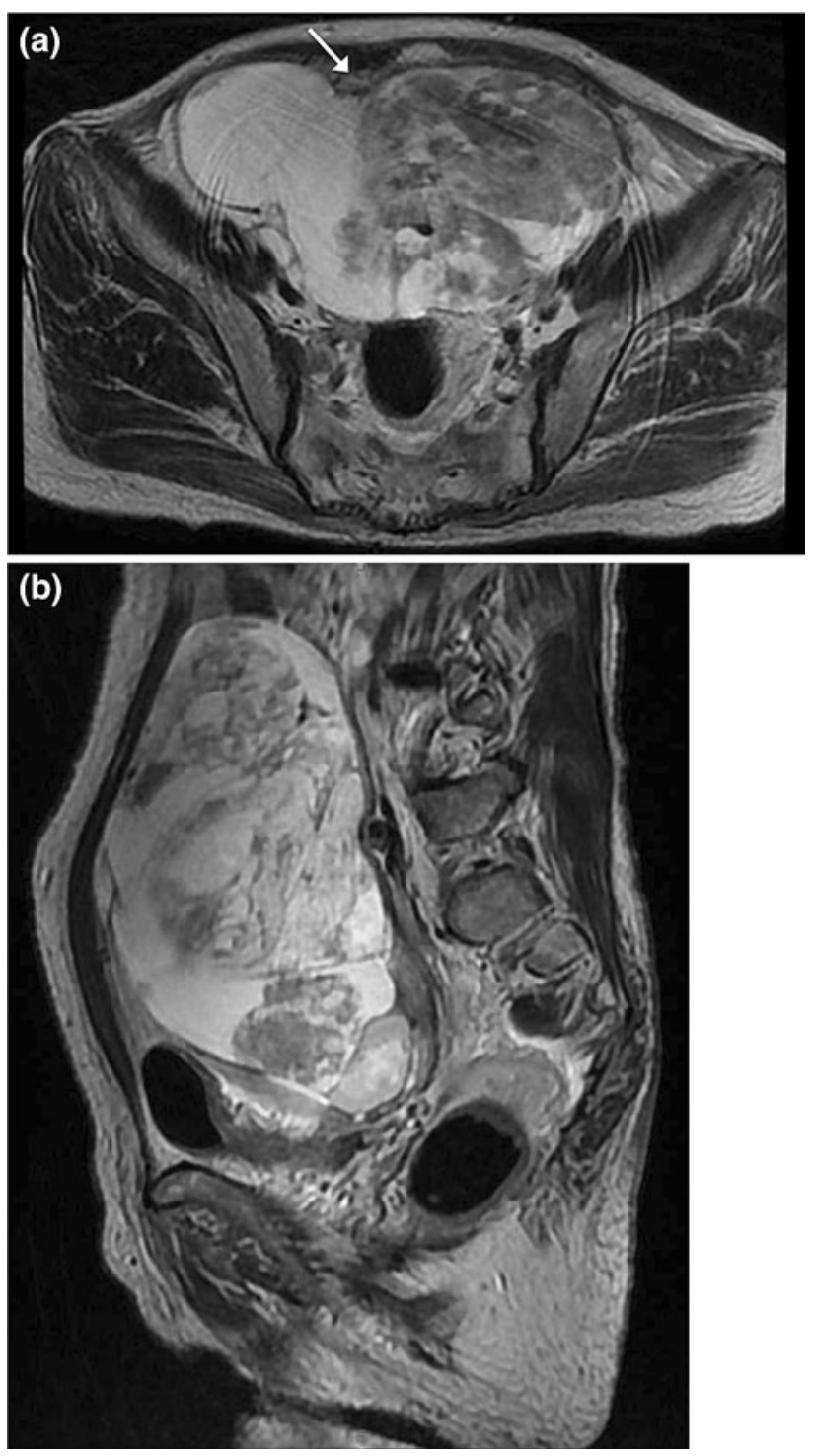

Fig. 4 a Axial and b sagittal T2-weighted pelvic MRI show a cystic and solid mass

Intravenous heparin was switched to warfarin to achieve a PT-INR of 2 to 3 . The patient received three cycles of postoperative adjuvant chemotherapy every 3 weeks with paclitaxel $\left(175 \mathrm{mg} / \mathrm{m}^{2}\right)$ and carboplatin $(\mathrm{AUC}=5)$. She has remained without evidence of recurrent cancer for 2 years after the completion of chemotherapy.

\section{Discussion}

Symptomatic VTE are associated with a risk of subsequent overt malignant disease [5-7, 13]. In a meta-analysis, overall, patients with VTE had a 3.2-fold risk of occult cancer. That risk varies according to tumor site; ovarian cancer is one of the highest risks. In the pooled cohort 
studies, relative risk (RR) for VTE as compared to no VTE was significantly elevated for ovarian cancer (RR 7.0) [1].

The molecular mechanisms inducing a prothrombotic state in patients with malignancy include tumoral production of procoagulants such as tissue factor (TF) and cancer procoagulant (CP) and inflammatory cytokines, and the interaction between tumor cells, blood, and endothelial cells. Other molecular mechanisms include some general responses of the host to the tumor, decreased levels of coagulation inhibitors, and impaired fibrinolysis [14]. As for ovarian cancer, TF expression is a possible determinant of VTE development. In particular, clear cell carcinoma may produce excessive levels of TF and is more likely to lead to the development of VTE [15].

Cerebrovascular events can also be the first manifestation of cancer. Among 5106 patients admitted for ischemic stroke, 24 patients $(0.4 \%)$ had an underlying malignancy. The pathogenetic pathways were nonbacterial thrombotic endocarditis (NBTE) (8/24), diffuse intravascular coagulation (DIC) (6/24), and atherosclerosis (5/24). Lung and breast cancer were most common malignancies. Two patients (8\%) had ovarian cancer [16]. Another case series showed that among 10 patients whose cancers were diagnosed at stroke presentation, one case $(10 \%)$ was ovarian cancer [17]. There were also some case reports in which stroke was the first manifestation of occult ovarian cancer. For this case report, we performed a comprehensive literature review and we summarize 8 cases, including the 2 cases in our institution, in Table 1 [9, 10, 16-18].

\section{Summary of eight cases}

Patients' ages at onset of stroke was from 37 to 62 , which is much younger than the average age cited in other epidemiological studies, where a quarter of all new strokes occur in people aged 65 years or older [19]. A histologic diagnosis of ovarian cancer was documented only in one case, except for our two cases of clear cell carcinoma. Histologically, clear cell carcinoma of the ovary has a different clinical behavior from other epithelial ovarian cancers. The incidence of VTE was significantly higher in patients with clear cell carcinoma than in patients with other epithelial ovarian cancers [2-4]. Although there was no clear pathological mechanism underlying the hypercoagulable state of a clear cell carcinoma, clear cell carcinoma may produce excessive levels of TF and is more likely to lead to the development of VTE [15]. Additionally, there were some reports about stroke due to NBTE associated with ovarian clear cell carcinoma $[11,12]$. Our cases are the first reported cases describing stroke as the first manifestation of ovarian clear cell carcinoma.

When our two cases developed strokes, our neurologist tried to determine an underlying etiology, but could not find underlying causes and mechanisms. Among five of the eight patients for whom the principle mechanisms of stroke pathogenesis were revealed, two patients developed NBTE, two DIC, and one patent foramen ovale (PFO). Neither of our two patients had DIC status, and there were no signs of PFO on echocardiography. We performed TTE, but because we did not perform transesophageal echocardiography (TEE) to make a definite diagnosis of NBTE, we may have missed that diagnosis. Despite the invasive nature of TEE, it is thought to be more sensitive in detecting valvular vegetations than TTE and is thus the preferred diagnostic test for this condition [20]. Although the possibility of NBTE in our two cases could not be ruled out, the other possible causes included atherosclerosis. Atherosclerosis is commonly related to the risk factors for stroke, which were none in case 1 , and diabetes and hypertension in case 2 . Taccone et al. [16] showed that $59 \%$ of patients in whom stroke is the first manifestation of an underlying malignancy had no risk factor, and $25 \%$ patients had at least 2 risk factors. In case 1, cancer-specific causes like NTBE are a reasonable mechanism of stroke rather than atherosclerosis.

For women with or without risk of cardiovascular disease, once cerebral infarction is diagnosed, they should undergo exhaustive evaluation for malignancy, particularly ovarian clear cell carcinoma.

Table 1 Review of the literature: stroke as the first manifestation of occult ovarian cancer

\begin{tabular}{lllllll}
\hline Authors & Year & Age & Histology & Stage & Duration from stroke to cancer & Stroke cause \\
\hline Borowski et al. [10] & 2005 & 46 & ND & IIIc & 7 months & NBTE \\
Wada et al. [18] & 2007 & 37 & Serous & Ic & 2 day & PFO \\
Yeh and Lin [9] & 2004 & 62 & ND & IIIc & ND & DIC \\
Taccone et al. [16] & 2008 & ND & ND & ND & ND & NBTE \\
& & ND & ND & ND & ND & DIC \\
Kwon et al. [17] & 2007 & 56 & ND & ND & 2 months & ND \\
Naoi et al. (this study) & 2013 & 55 & Clear & IIIc & 4 months & Unclear \\
& & 62 & Clear & Ic & 3 months & Unclear \\
\hline
\end{tabular}

ND not documented, NBTE nonbacterial thrombotic endocarditis, $P F O$ patent foramen ovale, DIC diffuse intravascular coagulation 
Conflict of interest The authors declare that they have no conflict of interest.

\section{References}

1. Iodice S, Gandini S, Löhr M et al (2008) Venous thromboembolic events and organ-specific occult cancers: a review and metaanalysis. J Thromb Haemost 6:781-788

2. Recio FO, Piver MS, Hempling RE et al (1996) Lack of improved survival plus increase in thromboembolic complications in patients with clear cell carcinoma of the ovary treated with platinum versus nonplatinum-based chemotherapy. Cancer 78:2157-2163

3. Goff BA, Sainz de la Cuesta R, Muntz HG et al (1996) Clear cell carcinoma of the ovary: a distinct histologic type with poor prognosis and resistance to platinum-based chemotherapy in stage III disease. Gynecol Oncol 60:412-417

4. Matsuura Y, Robertson G, Marsden DE et al (2007) Thromboembolic complications in patients with clear cell carcinoma of the ovary. Gynecol Oncol 104:406-410

5. Prandoni P, Lensing AW, Büller HR et al (1992) Deep-vein thrombosis and the incidence of subsequent symptomatic cancer. N Engl J Med 327:1128-1133

6. Baron JA, Gridley G, Weiderpass E et al (1998) Venous thromboembolism and cancer. Lancet 351:1077-1080

7. Sørensen HT, Mellemkjaer L, Steffensen FH et al (1998) The risk of a diagnosis of cancer after primary deep venous thrombosis or pulmonary embolism. N Engl J Med 338:1169-1173

8. Abrey LE, Dalmau JO (1999) Neurologic complications of ovarian carcinoma. Cancer 85:127-133

9. Yeh PS, Lin HJ (2004) Cerebrovascular complications in patients with malignancy: report of three cases and review of the literature. Acta Neurol Taiwan 13:34-38
10. Borowski A, Ghodsizad A, Gams E (2005) Stroke as a first manifestation of ovarian cancer. J Neurooncol 71:267-269

11. Aryana A, Esterbrooks DJ, Morris PC (2006) Nonbacterial thrombotic endocarditis with recurrent embolic events as manifestation of ovarian neoplasm. J Gen Intern Med 21:C12-C15

12. Devulapalli S, Pinto N, Gandothra C et al (2012) A rare case of occipital stroke as a consequence of nonbacterial thrombotic endocarditis in ovarian clear cell carcinoma: a case report. Case Rep Neurol 4:84-91

13. White $\mathrm{RH}$, Chew HK, Zhou $\mathrm{H}$ et al (2005) Incidence of venous thromboembolism in the year before the diagnosis of cancer in 528693 adults. Arch Intern Med 165:1782-1787

14. De Cicco M (2004) The prothrombotic state in cancer: pathogenic mechanisms. Crit Rev Oncol Hematol 50:187-196

15. Uno K, Homma S, Satoh T et al (2007) Tissue factor expression as a possible determinant of thromboembolism in ovarian cancer. Br J Cancer 96:290-295

16. Taccone FS, Jeangette SM, Blecic SA (2008) First-ever stroke as initial presentation of systemic cancer. J Stroke Cerebrovasc Dis 17:169-174

17. Kwon HM, Kang BS, Yoon BW (2007) Stroke as the first manifestation of concealed cancer. J Neurol Sci 258:80-83

18. Wada Y, Takahashi R, Yanagihara C et al (2007) Paradoxical cerebral embolism as the initial symptom in a patient with ovarian cancer. J Stroke Cerebrovasc Dis 16:88-90

19. Bonita R (1992) Epidemiology of stroke. Lancet 339:342-344

20. Lee RJ, Bartzokis T, Yeoh TK et al (1991) Enhanced detection of intracardiac sources of cerebral emboli by transesophageal echocardiography. Stroke 22:734-739 